\title{
A novel image cytometric method for quantitation of immunohistochemical staining of cytoplasmic antigens
}

\author{
M. Guillaud ${ }^{\mathrm{a}, *}$, J. B. Matthews ${ }^{\mathrm{a}}$, A. Harrison ${ }^{\mathrm{c}}$, C. MacAulay ${ }^{\mathrm{a}}$ and K. Skov ${ }^{\mathrm{b}}$ \\ ${ }^{\mathrm{a}}$ Cancer Imaging Department and ${ }^{\mathrm{b}}$ Medical Biophysics Department, BC Cancer Research Centre, \\ 601 West 10th Ave., Vancouver, BC, V5Z 1L3, Canada \\ ${ }^{\mathrm{c}}$ Oncometrics Imaging Corporation, Suite 505, 600 West Broadway, Vancouver, BC, \\ V5Z 1G1, Canada
}

Received 11 October 1996

Revised 1 April 1997

\begin{abstract}
Evaluation of molecular markers by immunohistochemical labelling of tissue sections has traditionally been performed by qualitative assessment by trained pathologists. For those markers with a staining component present outside of the nucleus, there has been no image histometric method available to reliably and consistently define cell interfaces within the tissue. We present a new method of approximating cellular boundaries to define cellular regions within which quantitative measurements of staining intensity may be made. The method is based upon Voronoi tessellation of a defined region of interest (ROI), and requires only the position of the nuclear centroids within the ROI.

Here we describe the VORSTAIN software which has been developed based on the Oncometrics CytoSavant Automated Image Cytometry System. To demonstrate this technique, human breast cancer sections immunohistochemically stained for $b c l-2$ protein and counter-stained with nuclear methyl green stain were evaluated. Intra-observer variation in the measured values was between $1.5-2.6 \%$ and inter-observer variation was between $1.8-4.4 \%$. The primary source of variability was due to difficulties in interpreting the exact position of the nuclear centroids. Analysis of mean staining densities for each slide correlated well with subjective scoring performed by two independent pathologists. Using VORSTAIN, significant variation of staining intensities between regions within the same slide was measured for some sections, indicating a large degree of heterogeneity within the tumours. The ability to accurately quantitate the degree of heterogeneity of molecular marker expression within tumours may be a valuable tool in prognostication.
\end{abstract}

Keywords: Quantitative immunohistochemistry, Voronoi diagrams, heterogeneity

\section{Introduction}

In general, most cancers are evaluated by morphologic and histologic criteria, and in many cases this information correlates with clinical outcome. However, there are many more cases where this information may be supplemented by evaluation of molecular markers, resulting in more accurate a priori determination of treatment response. These markers vary significantly in their function, relating to

\footnotetext{
${ }^{*}$ Corresponding author. Tel.: +1 6048776010 loc. 3099; fax: +1 6048756857 .
} 
proliferation, angiogenesis, malignant potential, etc. For instance in breast cancer, expression levels of the HER-2/neu oncogene product [21] and oestrogen and progesterone receptor levels [9], among others, have been shown to correlate with clinical outcome. It is now clear that in many cases such as these, patients may receive significant benefit from routine application of the information contained in these measurements $[18,22]$. As such, it is important that new techniques become available which allow the pathologist to perform these measurements easily and reproducibly. With recent advances in imaging technology, it is now possible to obtain quantitative information on a cell-by-cell basis from paraffin-embedded formalin-fixed tissue sections.

In the case of antigens which are localised within the cell nucleus, quantitation of immunostaining may be performed by image analysis using a technique known as "nuclear masking" [5]. Briefly, DNA is quantitatively stained (i.e., Feulgen staining) and the image of the stained material used to create a mask of the nuclear boundaries. The immunohistochemical signal is quantitated within these masks on a cell-by-cell basis and any influence from the DNA-stain removed by calibration techniques. This results in accurate and reproducible assessment of immunohistochemical staining, and has been demonstrated for $p 53$ [3], oestrogen receptors [5], and other markers [16].

In the case where there is an extra-nuclear staining component, analysis has traditionally been performed by qualitative assessment by a trained pathologist, resulting in a section-wide score describing the antigenic expression level [20]. Although this method is economical and time saving, it is highly subjective and results in much potential information being ignored. Imaging strategies have been devised which provide quantitative information regarding immunohistochemical staining, but suffer from several problems. Cytoplasmic boundaries may be manually defined using, for example, a computer pointing device, such that two-colour analysis may be performed based on these masks [23]. However, this is inappropriate in a clinical setting owing to the extreme time requirements. Another method which has been applied uses quantitative DNA staining to estimate the total number of cells within a field. Division of the total immunostain density within the field by the estimated number of cells results in an average staining intensity per cell [4]. However, any information regarding the variation in staining intensity is lost, although it is not known at this time whether such information is important.

In either case, image analysis of immunohistochemical staining with an extra-nuclear component has been difficult due to the lack of a simple, routine and reproducible method of segmenting the tissue. In the following work we describe a novel method of quantitation based on Voronoi tessellation of regions of interest within a tissue section, resulting in a consistent measurement of the heterogeneity of immunostaining. We demonstrate this technique using bcl-2 oncoprotein expression in breast tumours, as it represents an interesting problem owing to the numerous histologic structures in breast tissue.

\section{Voronoi diagrams}

The Voronoi diagram is a concept first described in 1850 by Dirichlet and subsequently refined by Voronoi in 1908 [2]. Although very well characterised, it has taken recent advances in computing power to develop this technique into useful applications. Thus, over the past decade a wealth of application have emerged in a number of scientific disciplines (i.e., crystallography, sociology, ecology, forestry, etc.).

Application of Voronoi diagrams to a set of points in a plane allows the partition of the space into polygonal regions. The edges of each polygon are the bisectors drawn between its associated point 


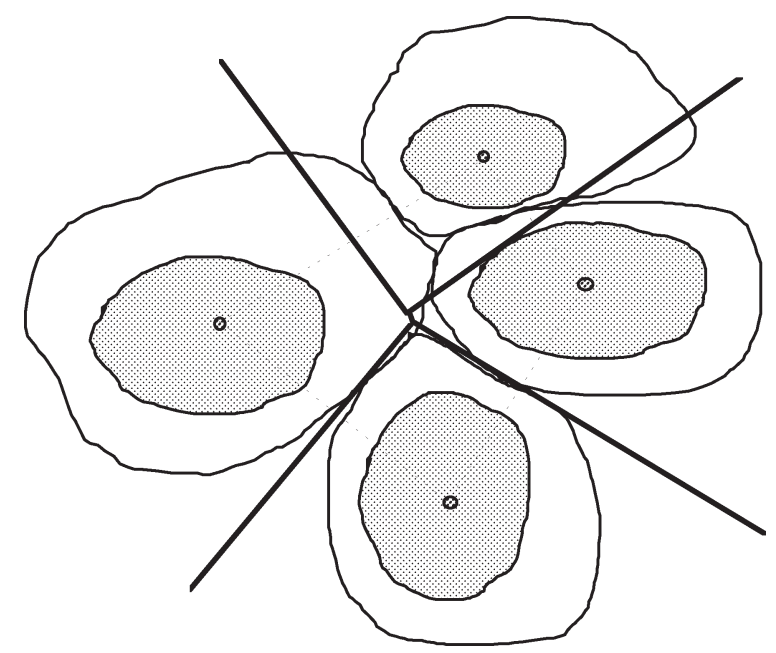

Fig. 1. Given a series of points, Voronoi polygons are constructed by drawing the bisectors of the lines joining each pair of adjacent points in a plane. The example shown here for four cells, illustrates how Voronoi polygons constructed based upon the centroids of cell nuclei can be used as an approximation of cellular boundaries. The bisectors, however, do not directly correspond to cell-cell interfaces.

and its neighbour, as is illustrated using the simple example in Fig. 1. The method of construction of these polygons is as follows.

Let $P=\left(p_{1}, p_{2}, \ldots, p_{n}\right)$ be a set of points in a two-dimensional Euclidean space, each point being referred to as a "site". Each co-ordinate within the plane (or within a specified region of interest) is then assigned to their nearest site, the set of co-ordinates thus forming the Voronoi region $V\left(p_{i}\right)$. Each region $V(i)$ therefore consists of all points at least as close to $p_{i}$ as to any other site. Those co-ordinates which have greater than one nearest site form the Voronoi diagram $V(P)$ for the set of sites $P . V(P)$ thus forms a paving of the space according to the positions of the $P$ sites, where each site has an associated Voronoi polygon in 2D which represents the area of influence of the site. Construction of the Voronoi diagram may be performed by brute force, however, it is typically constructed by application of an algorithm which uses successive addition of sites combined with only local modification of the diagram. This algorithm is described in detail elsewhere [7], but is illustrated schematically in Fig. 2. Typically, this algorithm is performed on a full microscope field, and marginal polygons are ignored. The algorithm has been modified such that marginal polygons were trimmed to regions of overlap with the ROI boundary in order that all polygons within the ROI could be analysed.

The prerequisite information for construction of Voronoi diagrams are thus simply a defined region of interest (ROI) and a series of sites within this region. In the case of histologic sections, it is customary to define the sites as the centroid of each cell nucleus. The resulting Voronoi diagram therefore contains information related to the inherent structure of the tissue and the notion of cellular neighbourhoods. This aspect of the method will not be further examined in this treatment, and the reader is referred elsewhere for further information $[6,8,17]$. Here, we choose to utilise the Voronoi polygons constructed from nuclear centroid positions to define cellular interfaces within the tissue. It must be noted, however, that by definition, construction of the set of Voronoi polygons requires only the position of the cell centroid and therefore cannot be viewed as a true segmentation method. Rather it should be viewed as a gross approximation of the cellular boundaries, as is illustrated schematically in Fig. 1. Other tessellation methods such as Laguerre's [1], utilise nuclear size to construct a series 


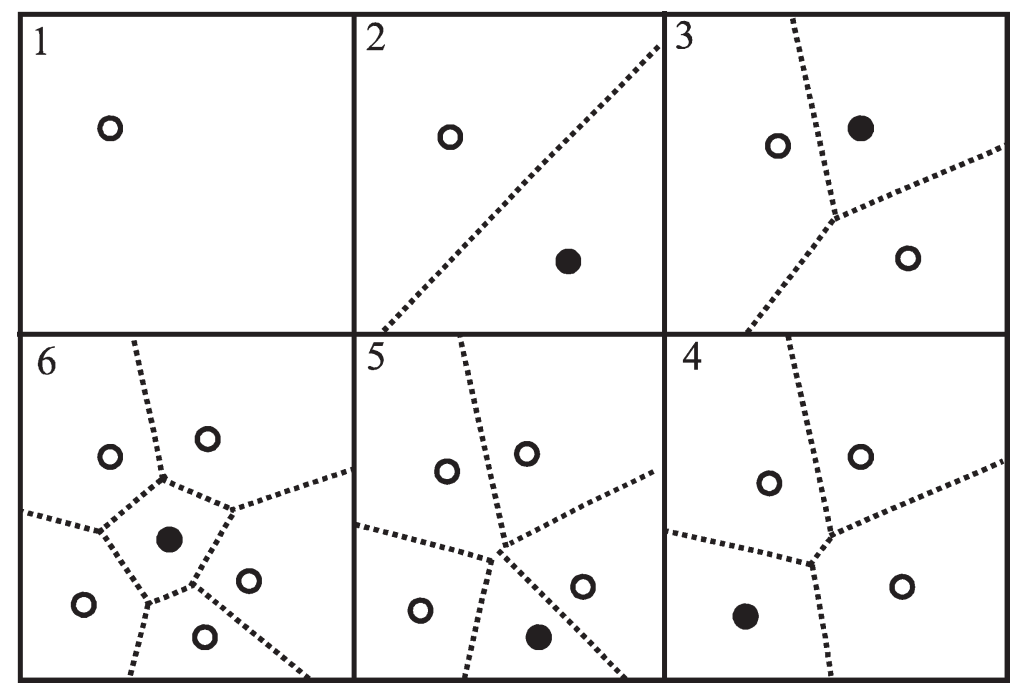

Fig. 2. Construction of a Voronoi diagram using successive addition of sites and local modification of the diagram. In each successively numbered frame, the solid circle represents the newly considered site. New bisectors are drawn between existing neighbour sites (open circles) and existing lines are cropped according to their relative position.

of cellular boundaries, however, this method assumes that there exists a constant ratio of nuclear and cytoplasmic cross-sectional area. Here we assume only that the nuclear centroid can be identified and that nuclear size is unknown and/or irrelevant. Each Voronoi polygon may be used to define a mask region approximating an individual cell's boundaries. To this end, we have developed interactive imaging software for analysis of biological stains with an extra-nuclear staining component.

\section{VORSTAIN interactive imaging software}

VORSTAIN is an interactive software which combines the image acquisition and processing capabilities of the CytoSavant image cytometry system (Oncometrics Imaging Corp., Vancouver, Canada) with Voronoi tessellation techniques. The CytoSavant is equipped with a 12-bit scientific CCD, a programmable shutter allowing user-control of integration time, a 8-bit to 12-bit look-up table (LUT) to control camera gain and a motorised stage programmable in the $x, y$ and $z$ directions. Further details pertaining to the CytoSavant have been described by Garner et al. [11]. Slides analysed with VORSTAIN must be stained so that the stain to be quantitated (with an extra-nuclear component, such as cytoplasmic antigens detected by immunohistochemical techniques) must be spectrally separable from the nuclear stain by the use of optical absorbance filters. For illustrative purposes, we assume here that all immunohistochemical staining is by DAB and DNA staining by methyl green such that $470 \pm 10 \mathrm{~nm}$ and $650 \pm 10 \mathrm{~nm}$ bandpassfilters are required. Slides are mounted on the microscope stage and the following steps are taken:

Initialisation. Two image channels are defined corresponding to both the nuclear stain and the stain of interest. Filters appropriate for each are placed in the light path and integration times and limits for LUT (effectively controlling the gain) are manually adjusted such that appropriate image brightness and contrast are obtained for each stain. The light levels remain constant through the rest of the procedure. A calibration image of a blank field is then captured using imaging parameters and a 
filter for the stain of interest. All further images of this stain are then calibrated by subtraction and normalised to the mode of this image to adjust for any illumination non-uniformities. The remaining steps in the procedure are described schematically in Fig. 3.

Region of interest selection. Using the imaging parameters and the filter for the nuclear stain, the slide may be manually scanned for a region of interest. Once found, an image is captured and the ROI may be defined interactively using one of several pre-defined structure-types: (1) epithelial, used for regions where the user defines two lines representing the basal lamina and the external membrane. The extremities of these lines are automatically joined; (2) ductal, used for annular regions where the user defines an outer ring representing the external membrane and an inner ring representing the lumen; and (3) other, where the user delineates a region of interest by contouring.

Extraction of nuclear centroids. Once the region of interest has been defined, nuclear centroids may be defined either manually or automatically. In this study, all nuclear centroids were manually extracted by interactive mouse-clicking on the centre of each nucleus within the ROI. Automatic extraction based on interactive thresholding of the nuclear image performed well but overlapping cell nuclei due to finite section thickness and overlapping of the DAB stain absorbance spectrum caused significantly higher imprecision than the manual method. The Voronoi diagram is then constructed based on the set of nuclear centroids and overlaid upon all further images.

Quantitation of the immunohistochemical staining. After switching filters to the $470 \mathrm{~nm}$ filter for imaging of $\mathrm{DAB}$ staining, another image using the previously defined parameters is acquired. Up to ten successive images of each polygonal area are taken in the $z$-plane $(0.5$ micron apart); the image with the highest variance in the pixel intensity histogram taken as the image of best focus. Labelling of each polygon is performed and cytometric features based on the DAB image are calculated for each. All cytometric features, images and polygonal overlays are then saved in a file. Following computation of the cytometric features for each polygon in the ROI, the user may repeat the procedure for any number of other ROIs.

File structures. Following the analysis of each ROI, cytometric features and images are saved on disk. A separate file is created which contains the number of ROIs analysed, their type and stage co-ordinates and pathology. Table 1 contains the complete list of information stored for each slide.

Analysis of cytometric features may be performed from within the VORSTAIN program which, like the CytoSavant image acquisition program allows the user to display features in both scatterplot and histogram formats, with features means, standard deviations and coefficients of variation for each cell type calculated for the selected features.

The VORSTAIN program also allows the import and export of images coupled to their slide coordinates. In this fashion, regions of interest may be identified by a pathologist and subsequently revisited on the microscope by a technician for analysis.

The measurement of immunohistochemical staining using VORSTAIN was tested using $5 \mu \mathrm{m}$ thick sections of primary breast carcinoma. Immunohistochemical labelling of the $b c l-2$ protein was performed according to Krajewski et al. [12,13] and subsequent counter staining with methyl green nuclear stain (Fig. 4). 


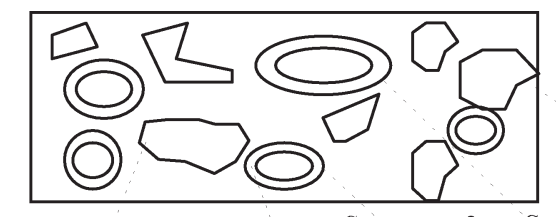

Double stained Breast biopsy section

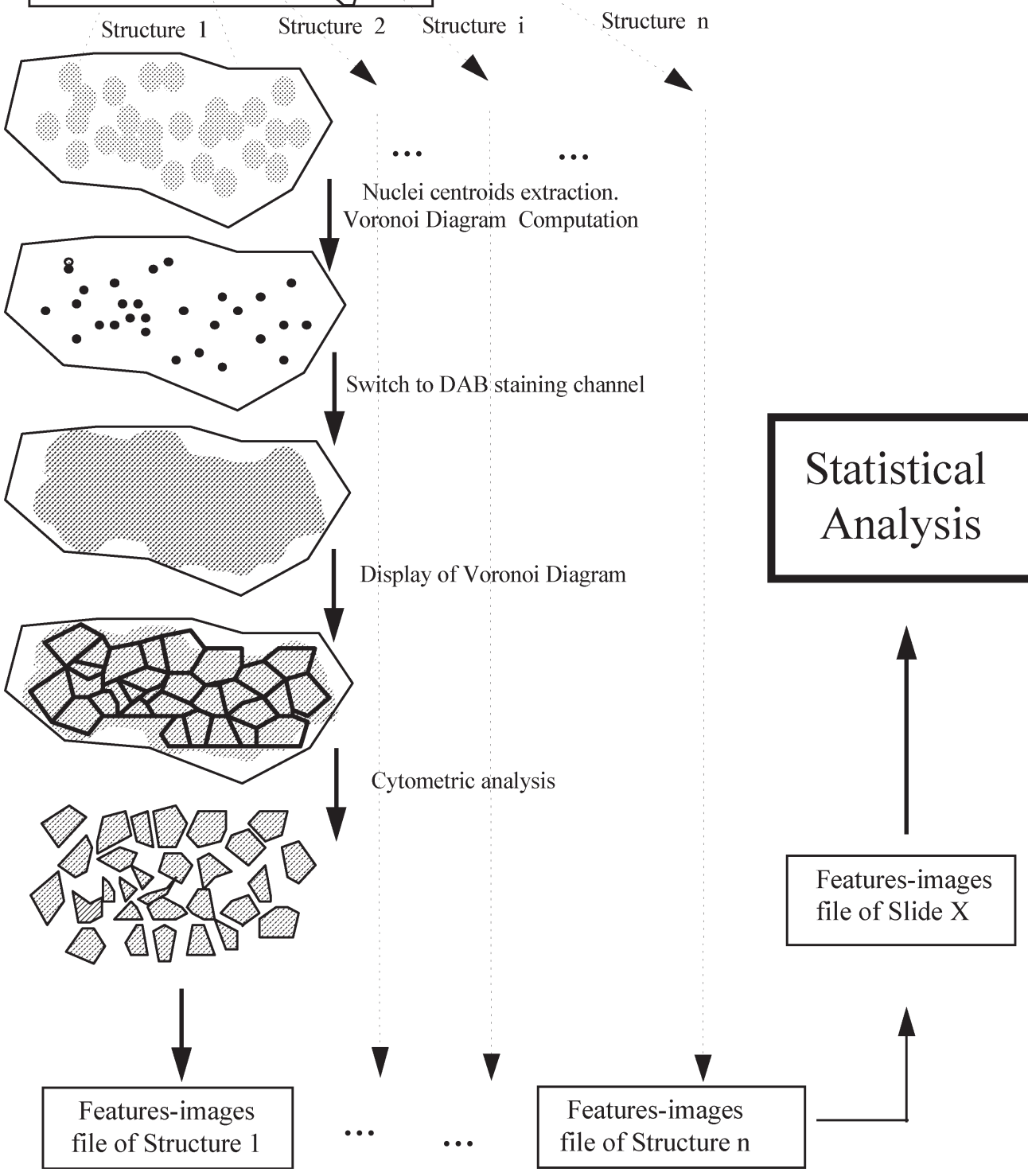

Fig. 3. A schematic illustration of the use of the VORSTAIN program. Several different structures may be identified on each slide, and grouped according to their histological type and position on the slide. For each structure, an image of the nuclear stain is acquired and used to identify the position of nuclear centroids within the ROI. An image of the immunohistochemical label is acquired and construction of the Voronoi diagram proceeds according to methods outlined in the text. Cytometric feature analysis is performed for each Voronoi polygon within the region and image/mask pairs and feature data are stored within a file, and analysis of another region of interest may begin. Statistical analysis of the features/images file may be performed at a later time. 
Table 1

Information from the analysis of each slide is stored in two files. The first (file extension '.img') contains cell images, polygonal masks and cytometric features. The second contains information specific to the Voronoi diagram, slide co-ordinates and pathology, with the '.cog' extension
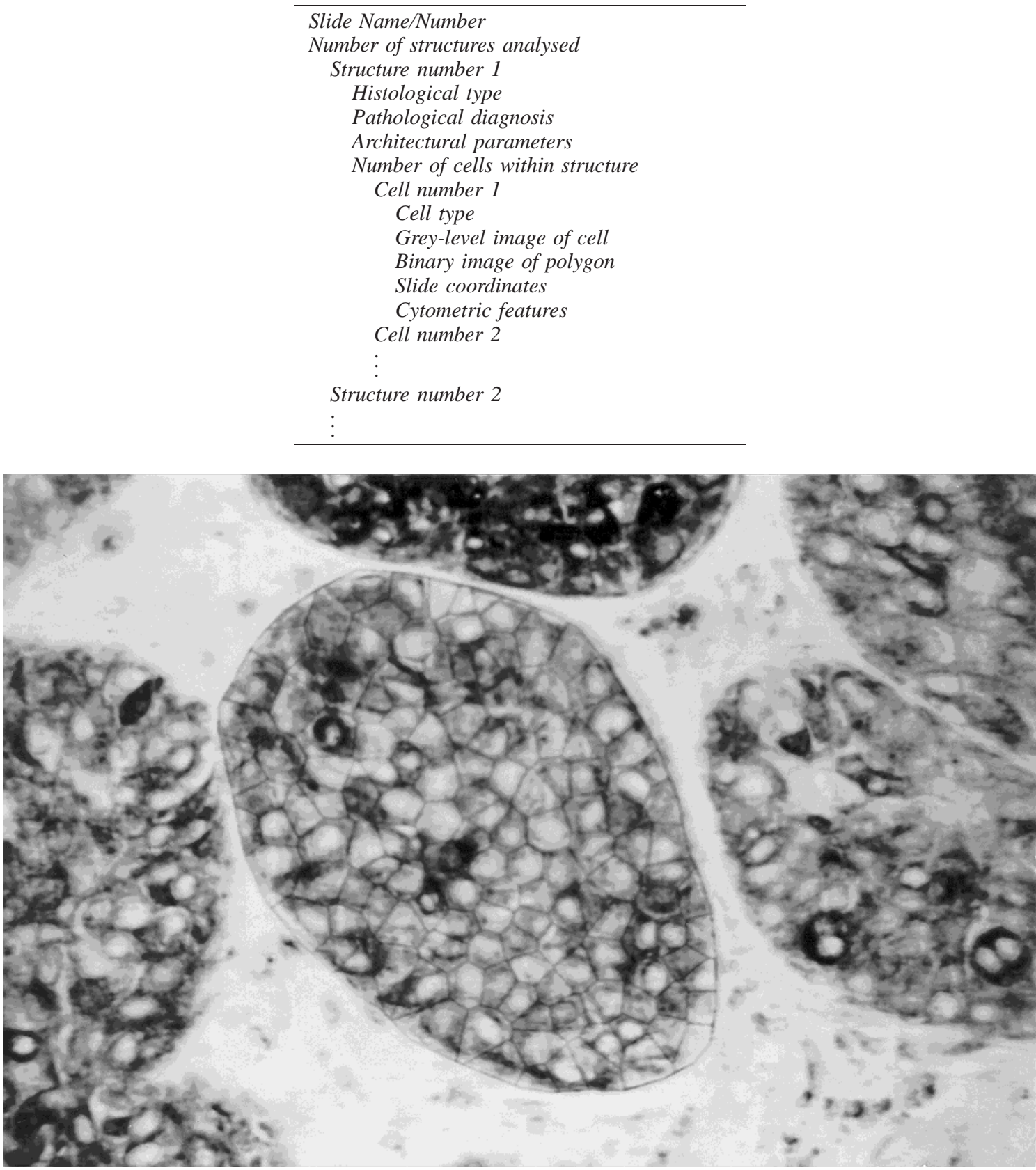

Fig. 4. Image acquired with VORSTAIN, demonstrating Voronoi tessellation of a region of DCIS immunohistochemically labelled for $b c l-2$ oncoprotein. 
Reproducibility of measurement. Three regions of Ductal Carcinoma In Situ (DCIS) of the breast were selected from a slide which had been previously classified as intensely stained, but had significant variations between regions such that areas of low, moderate and intense staining could be visually identified. The light level was adjusted prior to measurements such that the mode of the pixel intensity distribution of a field acquired from a blank, coverslipped slide was 226, in order to ensure field illumination between sessions remained constant. For confirmation, a sample of 100 Feulgen-thionin stained human peripheral blood lymphocytes was acquired (according to Garner et al. [11]) at the beginning of each session, the mean DNA amount recorded as confirmation that the light level was consistent.

All statistical analysis was performed on a PC using Statistica (Statsoft Inc., Tulsa, Oklahoma).

\section{Results}

Table 2 lists results for the three chosen regions. The integrated optical density (IOD) for each Voronoi polygon within the ROIs was normalised by the polygonal area to give the area normalised IOD (IOD/area) for each polygon. One-way ANOVA performed on the mean IOD/area distributions for five independent measurements of each ROI showed that the measurements gave statistically similar results $(p>0.05)$ with coefficients of variation (c.v.) ranging from 1.5 to $2.6 \%$. Similarly, ANOVA

\section{Table 2}

Reproducibility measurements performed on DCIS components of a primary breast carcinoma. Of each ROI, the number of cells, mean polygon area, mean IOD/area and standard deviation of IOD/area are listed. Intra-observer c.v. is shown for each area. $p$ values from one-way ANOVA performed on five repeated measurements on each of three separate regions within the slide indicated that no significant differences between distributions of area normalised IOD (IOD/area) existed. A single region (denoted 3299a and 3299a*) was measured by two independent observers, who were also tested and found to be statistically the same

\begin{tabular}{|c|c|c|c|c|c|c|c|c|}
\hline Region & Trial & $\#$ of cells & Mean area & $\mathrm{CV}$ area & $\begin{array}{c}\text { Mean IOD/ } \\
\text { area }\end{array}$ & $\begin{array}{l}\text { Stand. dev. } \\
\text { IOD/area }\end{array}$ & $p$ & $\begin{array}{c}p \\
\text { (inter-observer) } \\
\end{array}$ \\
\hline \multirow[t]{5}{*}{$3299 a$} & 1 & 282 & 1268 & & 0.1327 & 0.0936 & & \multirow{20}{*}{0.1057} \\
\hline & 2 & 304 & 1170 & & 0.1396 & 0.0979 & & \\
\hline & 3 & 281 & 1260 & 0.057 & 0.1365 & 0.0950 & 0.9194 & \\
\hline & 4 & 259 & 1374 & & 0.1385 & 0.0972 & & \\
\hline & 5 & 284 & 1264 & & 0.1387 & 0.0958 & & \\
\hline \multirow{5}{*}{ 3299a* } & 1 & 312 & 1165 & \multirow{5}{*}{0.076} & 0.1328 & 0.0984 & \multirow{5}{*}{0.9444} & \\
\hline & 2 & 287 & 1280 & & 0.1338 & 0.0920 & & \\
\hline & 3 & 347 & 1053 & & 0.1379 & 0.1028 & & \\
\hline & 4 & 295 & 1250 & & 0.1330 & 0.0982 & & \\
\hline & 5 & 293 & 1245 & & 0.1344 & 0.0937 & & \\
\hline \multirow[t]{5}{*}{$3299 b$} & 1 & 254 & 1897 & \multirow{5}{*}{0.013} & 0.0117 & 0.0139 & \multirow{5}{*}{0.9778} & \\
\hline & 2 & 251 & 1937 & & 0.0116 & 0.0145 & & \\
\hline & 3 & 255 & 1914 & & 0.0111 & 0.0147 & & \\
\hline & 4 & 254 & 1902 & & 0.0119 & 0.0151 & & \\
\hline & 5 & 258 & 1869 & & 0.0116 & 0.0149 & & \\
\hline \multirow[t]{5}{*}{$3299 \mathrm{c}$} & 1 & 340 & 1423 & \multirow{5}{*}{0.019} & 0.1444 & 0.0872 & \multirow{5}{*}{0.6650} & \\
\hline & 2 & 339 & 1421 & & 0.1409 & 0.0869 & & \\
\hline & 3 & 347 & 1399 & & 0.1469 & 0.0920 & & \\
\hline & 4 & 359 & 1354 & & 0.1509 & 0.0959 & & \\
\hline & 5 & 345 & 1404 & & 0.1482 & 0.0916 & & \\
\hline
\end{tabular}


performed on five repeated measurements of a single ROI by each of two independent observers showed a non-statistically significant difference $(p>0.05)$ and c.v.'s were $1.8-4.4 \%$. Measurements of IOD alone (not normalised by polygon area) were statistically similar $(p>0.05)$ when performed by a single observer, however, a significant difference was found for inter-observer measurements (data not shown, $p<0.05$ ). ANOVA performed on mean IOD between different ROIs on measurements by a single observer showed that these were statistically different regions $(p \leqslant 0.05)$.

Correlation with bcl-2 score. A set of five slides was chosen which reflected the range of values assigned by objective scoring by two independent pathologists. Since it is typically the invasive component of the carcinoma which is scored, measurements were performed only on ROIs consisting entirely of invasive carcinoma cells. Slides had been previously evaluated on a scale from 0 to 3 ( 0 none; 1 - mild; 2 - moderate; 3 - intensely stained). Of the slides obtained, only one slide had been scored as 0 , but too few cells were identifiable to perform measurements. Similarly, only a single slide of score ' 1 ' was obtained, and only two fields of invasive carcinoma were visible. For slides scored as ' 2 ' and ' 3 ', three regions of invasive carcinoma each were randomly chosen for study. Due to the fragmented nature of the invasive regions, measurements sometimes had to be performed by delineating several ROIs within a single microscope field. Data was gathered such that images of approximately 100 cells per field were acquired. Figure 5 shows the relationship between the mean IOD/area and the pathologists' score. Each point represents the mean of a single microscope field (or ROI) and different slides are represented by different icons. A linear relationship between the mean IOD/area and the objective score can be drawn by averaging measurements of regions from slides with similar subjective grading (as shown, $r=0.9995$ ). Similarly, there was an approximately linear relationship between the highest mean score per slide (as shown, $r=0.9998$ ). There was considerable intra-slide variation in staining intensity observed in several of the cases. The intra-region (i.e., interpolygonal) variation in staining intensity was found to increase with pathologists' score in a manner

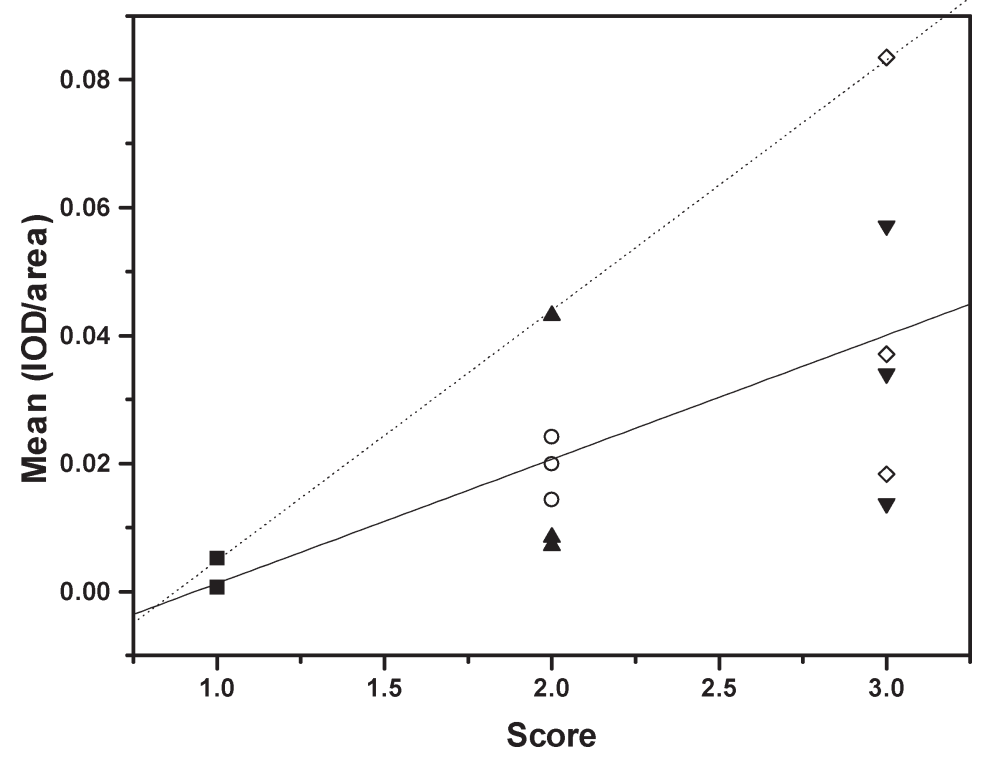

Fig. 5. Relationship between IOD/area and pathologist score for $b c l-2$ protein expression. Each point represents the mean of approximately 100 cells analysed from a single microscope field. Five slides were analysed, with up to three regions chosen for each. The dashed line is drawn through the highest mean score per slide and a solid line is drawn through the slide means. 


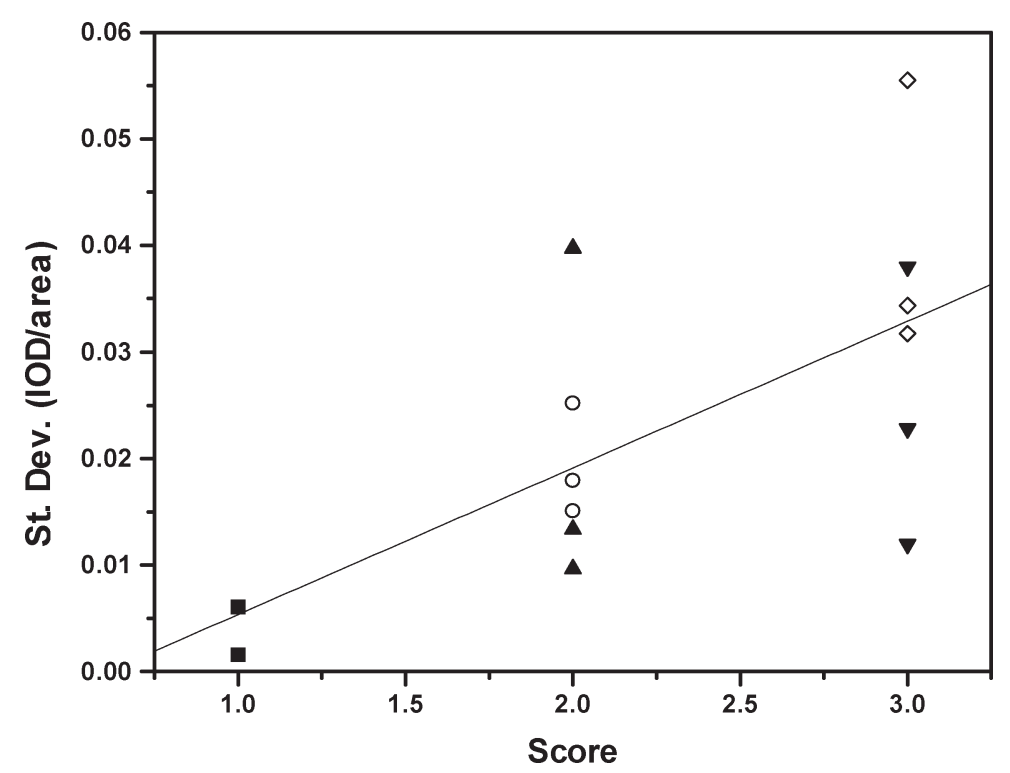

Fig. 6. Relationship between standard deviation of IOD/area and pathologist score for $b c l-2$ protein expression for the same regions shown in Fig. 4. A line is drawn through the slide mean values.

strikingly similar to the mean IOD/area, as demonstrated in Fig. 6. The average standard deviation of scoring also increased linearly with pathologists' score $(r=0.9998)$.

\section{Discussion}

These studies demonstrate that application of Voronoi diagrams to delineate cellular interfaces could be a useful tool in quantitative analysis of immunohistochemical staining. The VORSTAIN system described here provides an interactive tool for research requiring the assessment of both the intensity and heterogeneity of immunolabelling procedures. While this system in no way pretends to be capable of accurately delineating cytoplasmic boundaries within tissue sections, it provides an architecturalbased method of tessellating a region of interest into cellular neighbourhoods, dependent on the size of the ROI and number and position of nuclei within the ROI.

Comparison of measurements performed with VORSTAIN highlight the subjective nature of routine pathological analysis of immunohistochemical staining levels. Significant intra-tumoural variations in staining density was found in several of the samples, yet this feature is typically ignored when global scoring methods are used. Although it is not currently accepted that such information is clinically useful, there is evidence in some cases that heterogeneity is responsible for treatment failure $[14,15]$, and in others that the pattern of expression of some proteins may be more relevant than the mean staining levels themselves [19]. VORSTAIN will also allow analysis of expression patterns post hoc, as all necessary information to analyse site-specificity of staining is recorded, including stage position, ROI co-ordinates, Voronoi structures, etc.

Since there is little information regarding intra-tumoural heterogeneity of immunohistochemical markers, it is unknown at this time what sample sizes will be necessary to correctly characterise individual tumours. It is surmised that this will be largely dependent on the nature of the label to be detected. For instance it may be envisioned that in the case of some labels, only the maximum staining 
intensity (or over-expression of a specific protein) would be of clinical value in a prognostic sense, and thus sampling could be restricted to those very intensely staining areas in a tumour. However, it may also be postulated that for some proteins it is the degree of heterogeneity itself which might be related to biological aggressiveness. For example in Fig. 2, two slides analysed were scored as '2' by pathological examination, but express very different levels of heterogeneity based on a sample of three regions. Studies are currently in progress to determine the number of ROIs and mean sample size necessary to achieve a stable measure of intra-tumoural heterogeneity. This analysis is expected to be specific to the marker under consideration and is being carried out for a number of proteins including $b c l-2$.

The reproducibility studies highlight that measurements of the distribution of IOD measurements, when normalised for polygonal area are highly reproducible. Coefficients of variation for the mean values obtained by five separate measurements of each of four ROIs were in the range of 0.015 0.026. Furthermore, measurements of intra-region heterogeneity were also highly reproducible, such that the standard deviations of the IOD/area histograms varied by only $1.8-4.4 \%$ over five trials. Thus, this system allows one to measure the global average staining intensity, the intra-slide heterogeneity and the intra-regional variation in staining intensity in a consistent and objective manner. While measurements of raw staining intensity (not normalised by polygonal area) were found to be slightly more inconsistent between observers, this may be attributed to several factors.

The data have been presented as IOD/area, since we found a not wholly unexpected correlation between polygonal IOD and polygonal area. Considering the three-dimensional nature of tissue sections and the possible inclusion of several layers of cells within a single polygon, this was deemed justified. However, normalisation of the IOD by polygonal area produces several theoretical and technical benefits over reporting raw IOD. The most significant variation occurs due to difficulties in identification of nuclear centroids. Some overlap in the spectra of the two stains meaning that there was a small DAB component within the image used for identification of centroids and there were frequent problems in discerning whether one was looking at a single oblong-shaped cell or two separate and overlapping nuclei. In addition, in many cases there were small portions of nuclei sheared during sectioning that were easily missed. Indeed, the number of cells within an ROI varied up to $20 \%$ in the cases described herein (Table 2). Because the boundaries of the ROIs may be clearly delineated by comparison, the total area of the ROI remained relatively constant between measurements. Therefore, the number of cells identified within a given ROI is inversely correlated with both the mean IOD for the ROI and mean area of individual polygons, as may be noted in Table 2. Moreover, it is the nature of Voronoi polygons that regions equidistant between two or more different sites are not associated exclusively with any one nucleus. These boundary points are used solely for delineation and are not included in the calculation of IOD. Therefore, a greater number of sites would result in a larger number of pixels used to define the polygons and therefore a lower IOD summed over the whole ROI. Division of the raw IOD by polygonal area circumvents the preceding problems, as may be seen in Table 2, where there is no consistent variation in mean IOD/area with cell number. The authors recognise that measurements of raw IOD may prove useful in the future and are taking steps to resolve this problem. Routines will be incorporated to subtract overlapping DAB signals from the image used for nuclear centroid identification and future plans include the evaluation and testing of various nuclear segmentation routines to more consistently develop a map of nuclear positions prior to Voronoi construction.

It should be noted, however, that quantitation of immunohistochemical staining is highly dependent on tissue preparation and staining methods. Strictly quantitative measurements can only be achieved 
when appropriate steps in quality control are taken and therefore may only be applicable to fresh biopsied samples. For archival tissue samples, or where quality control procedures are lacking, it may be possible to obtain semi-quantitative assessment of tumour markers using VORSTAIN, if data can be scaled according to IOD measurements from internal positive and negative controls. Furthermore, the application of this technique may prove more useful for fluorescence detection of immunuhistochemical staining. The higher degree of linearity between stain intensity and amount of antigen afforded by fluorescence techniques combined with the improved ability to separate two stains spectrally makes this approach more attractive than absorbance methods.

In summary, the application of Voronoi diagrams to quantitative measurement of immunohistochemical staining opens exciting possibilities for the study of prognostic markers. It allows objective measurement of cytoplasmic (and other) expressed tumour markers and provides a reproducible method for the assessment of the relationship of tumour heterogeneity to the biological aggressiveness of tumours.

\section{Acknowledgements}

The authors wish to thank Dr G. Bebb and Dr M. Hayes of the BC Cancer Agency for pathological diagnosis of tissue sections and would like to acknowledge the kind support of Dr J. Reed and Dr S. Krajewski of the La Jolla Cancer Research Foundation for immunohistochemical labelling of the sections. Thanks are also extended to Dr K. Gelmon of the BC Cancer Agency for her thoughtful discussions. This work was performed with the financial support of the Lohn Foundation of British Columbia, the BC Chapter of the Canadian Breast Cancer Research Foundation and the Science Council of BC.

\section{References}

[1] F. Aurenhammer, Power diagrams: Properties, algorithms and applications, SIAM J. Comput. 16 (1987), 78-96.

[2] F. Aurenhammer, Voronoi diagrams: a survey of fundamental data structure, ACM Comput. Surveys 23 (1991), 345-405.

[3] B.C. Aziz and R.B. Barathur, Quantitation and morphometric analysis of tumours image analysis, J. Cell Biochem. 19 (1994), 120-125.

[4] S.S. Bacus, M.F. Press, J.W. Bacus and D.J. Slamon, HER-2/neu oncogene expression and DNA ploidy analysis in breast cancer, Arch. Pathol. Lab. Med. 114 (1990), 164-169.

[5] S. Bacus, J.L. Flowers, M.F. Press, J.W. Bacus and K.S. McCarty, The evaluation of estrogen receptor in primary breast carcinoma by computer-assisted image analysis, Am. J. Clin. Pathol. 90 (1988), 233-239.

[6] G. Bigras, R. Marcelpoil, E. Brambilla and G. Brugal, Cellular sociology applied to neuroendocrine tumours of the lung: quantitative model of neoplastic architecture, Cytometry 24 (1996), 74-82.

[7] A. Bowyer, Computing Dirichlet tessellation, Comput. J. 24 (1981), 162-166.

[8] R. Chandebois, Cell sociology: a way of reconsidering the current concepts of morphogenesis, Acta Bioth. (Netherlands) 25 (1976), 71-102.

[9] B. Chevallier, F. Heintzman, V. Mosseri, J.P. Dauce, P. Bastit, Y. Graic, P. Brunelle, J.P. Basuyau, M. Comoz and B. Asselain, Prognostic value of oestrogen and progesterone receptors in operable breast cancer. Results of a univariate and multivariate analysis, Cancer 62 (1988), 2517-2524.

[10] P.J. van Diest, J.P.A. Baak, D. Chin, J.W. Theeuwes and S.S. Bacus, Quantitation of HER-2/neu oncoprotein overexpression in invasive breast cancer by image analysis: a study comparing fresh and paraffin embedded material, Analyt. Cell. Pathol. 3 (1991), 195-202.

[11] D.M. Garner, A. Harrison, C. MacAulay and B. Palcic, CytoSavant (tm) and its use in automated screening of cervical smears, in: Compendium on the Computerized Cytology and Histology Laboratory, G.L. Wied, P.H. Bartels, D.L. Rosenthal and Schenck, eds, Tutorials of Cytology, Chicago, 1994, pp. 346-352.

[12] S. Krajewski et al., Immunohistochemical analysis of $m c l-1$ and $b c l-2$ proteins in normal and neoplastic lymph nodes, Am. J. Pathol. 145 (1994), 515-525. 
[13] S. Krajewski et al., Immunohistochemical analysis of $m c l-1$ protein in human tissues: differential regulation of $m c l-1$ and $b c l-2$ protein production suggests a unique role for $m c l-1$ in control of programmed cell death in vivo, Am. J. Pathol. 146 (1995), 1309-1319.

[14] M.E. Lippman and J.C. Allegra, Estrogen receptor and endocrine therapy of breast cancer, N. Engl. J. Med. 299 (1978), 930-933.

[15] H. Maass, W. Jonat, G. Stolzenbach and G. Trams, The problem of non-responding estrogen receptor positive patients with advanced breast cancer, Cancer 46 (1980), 2835-2837.

[16] S.V. Makkink-Nombrado, J.P.A. Baak, L. Schuurmans, J.W. Theeuwes and T. van der Aa, Quantitative immunohistochemistry using the CAS 200/486 image analysis system in invasive breast carcinoma: a reproducibility study, Anal. Cell. Pathol. 8 (1995), 227-245.

[17] R. Marcelpoil and Y. Usson, Methods for the study of cellular sociology: Voronoi diagrams and parameterization of the spatial relationships, J. Theor. Biol. 154 (1992), 359-369.

[18] R.J. Pamies and D.R. Crawford, Tumour markers. An update, Med. Clin. N. Amer. 80 (1996), 185-99.

[19] T. Pietilainen, P. Lipponen, S. Aaltomaa, M. Eskelinen, V.M. Kosma and K. Syrjanen, Expression of $c-m y c$ proteins in breast cancer as related to established prognostic factors and survival, Anticancer Res. 15 (1995), 959-964.

[20] D.S. Schultz, R.L. Katz, S. Patel, D. Johnson and N.G. Ordonez, Comparison of Visual and CAS-200 quantitation of immunocytochemical staining in breast carcinoma samples, Analyt. Quant. Cytol. Histol. 14 (1992), 35-40.

[21] D.J. Slamon, G.M. Clark, S.G. Wong, W.J. Levin, A. Ullrich and W.L. McGuire, Human breast cancer: correlation with relapse and survival with amplification of the HER-2/neu oncogene, Science 235 (1987), 177-182.

[22] G.W. Sledge, Implications of the new biology for therapy in breast cancer, Semin. Oncology 23 (1996), 76-81. 


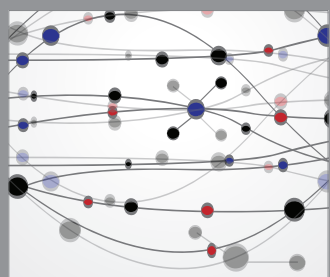

The Scientific World Journal
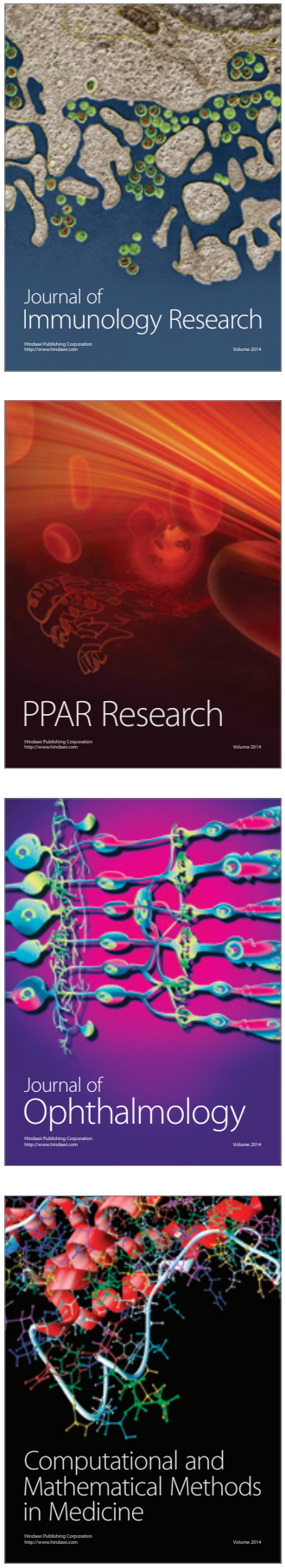

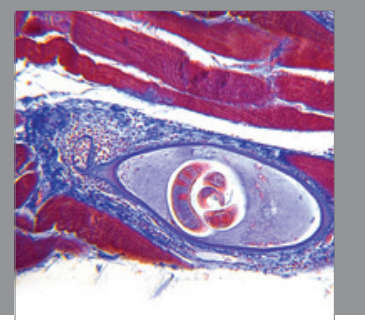

Gastroenterology

Research and Practice
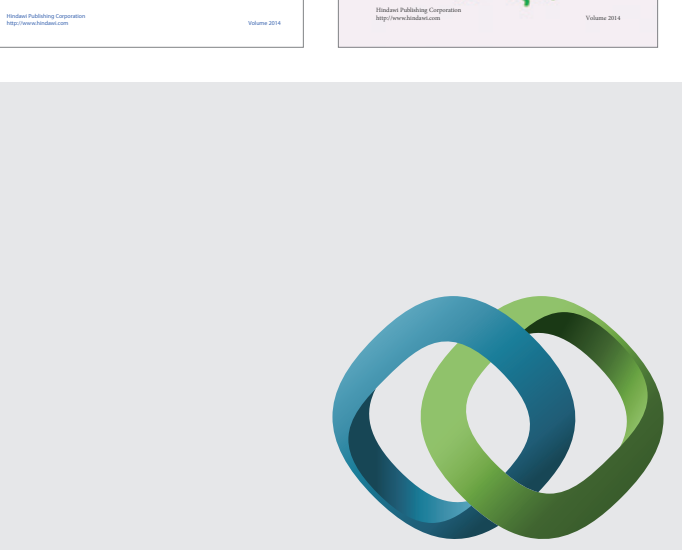

\section{Hindawi}

Submit your manuscripts at

http://www.hindawi.com
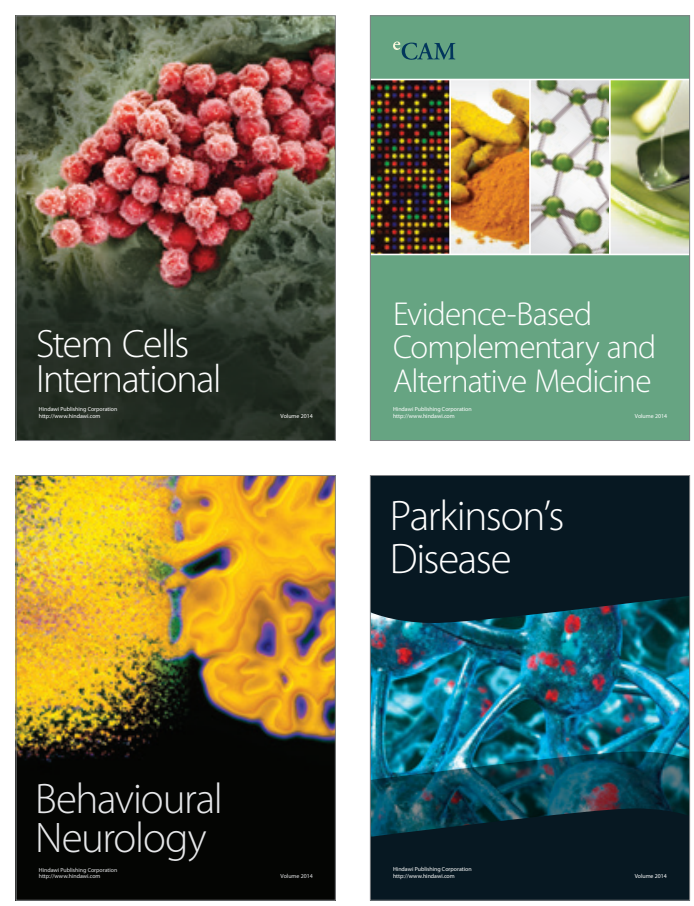

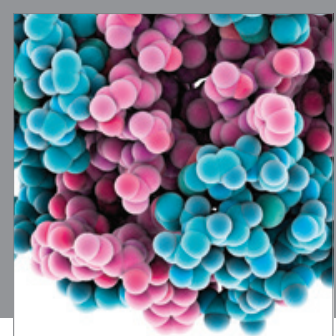

Journal of
Diabetes Research

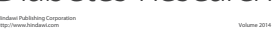

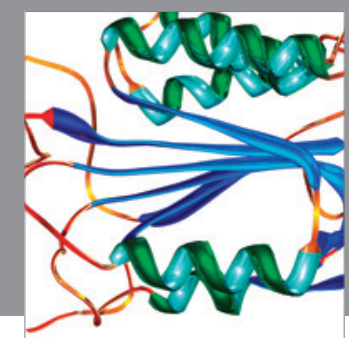

Disease Markers
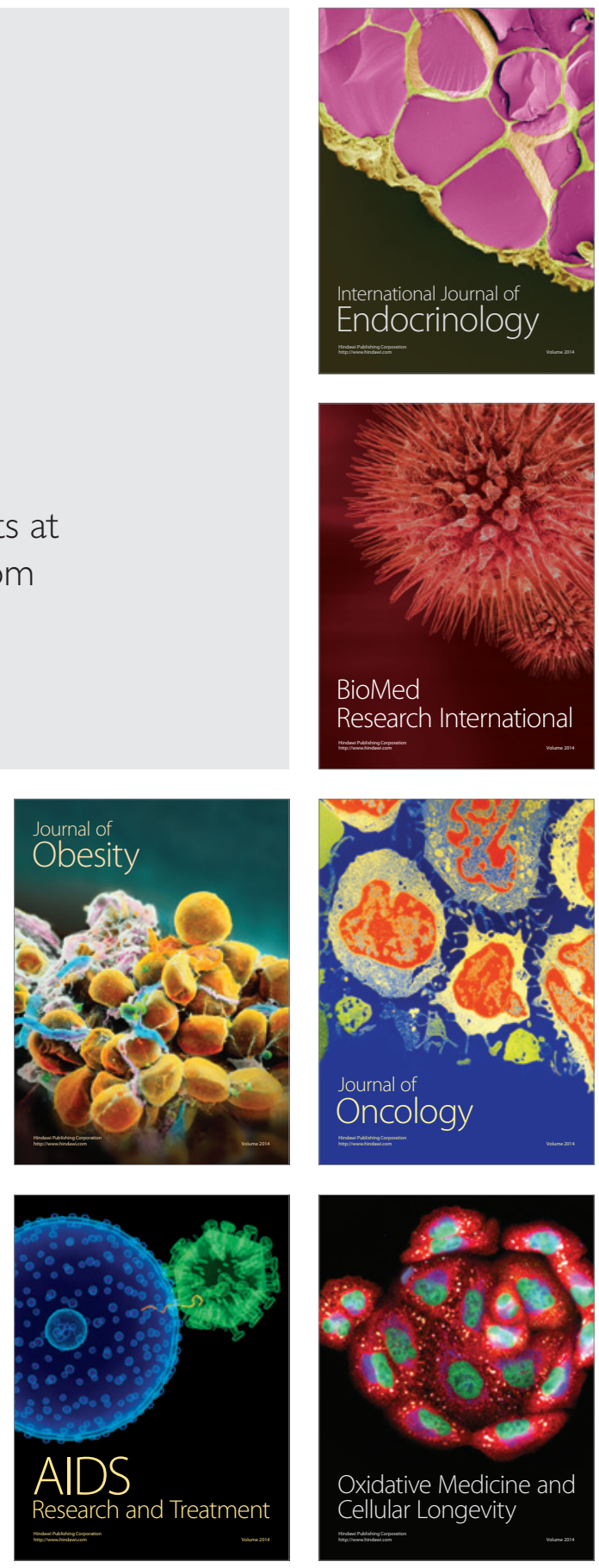